Hunt's story reaches its climax at the momentous 1888 meeting of the British Association in Bath. It was here that FitzGerald made the sensational announcement that the German physicist Heinrich Hertz had succeeded in generating and detecting electromagnetic waves. The widespread excitement produced by Hertz's experiments brought Maxwell's Treatise to the fore in physics and further cemented the growing friendship between its leading British interpreters. During the six years that followed - a period referred to by Hunt as the "Maxwellian heyday" - FitzGerald, Lodge and Heaviside worked in close collaboration to popularize and establish Maxwellian electromagnetic theory. One of the many pieces of Maxwellian physics from this period discussed by Hunt is the now infamous 'contraction hypothesis' proposed by FitzGerald in 1889 to account for the null result of the Michelson-Morley etherdrift experiment. The sensitive documentation of this much abused episode in the history of physics is one of the high points of the book, and should finally lay to rest discussion of the influence of this hypothesis in Einstein's subsequent work on relativity.

The Maxwellians is a remarkable achievement for several reasons. First, Hunt is completely familiar with the enormous archive (correspondence and manuscripts) of the Maxwellians; yet he manages to do justice to this literature without overly frequent, or overly long, quotation from his sources. Second, the book is exemplary of the recent trend among historians of science towards placing scientific achievements in a broader historical context. As Hunt makes clear, Maxwellian electromagnetic theory was intimately linked to the Victorian culture from which it sprang and cannot be understood in isolation. Finally, and perhaps most impressively, Hunt combines the highest level of professional historical scholarship with a narrative that is lively and compelling throughout. The only technical material over which a reader without a degree in physical sciences might stumble (Heaviside's route to 'Maxwell's equations') is conveniently relegated to an appendix. These considerable virtues make the book compulsory reading for the professional historian of modern science, and invaluable (and very welcome) as a teaching aid for students working in the field. Anyone interested in the history of physics or electrical engineering will surely find it fascinating.

Andrew Wanwick is in the Department of History and Philosophy of Science, University of Cambridge, Cambridge CB2 $3 R H$, UK.

\title{
Chemical cookbook
}
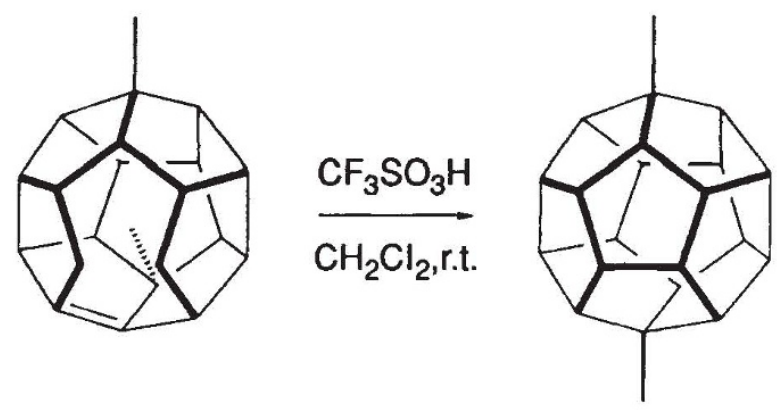

Recipes for putting together just about any organic molecule from the simplest alkene to the extravagant edifice of dodecahedrane (a derivative of which is pictured here) can be found in the nine-volume series Comprehensive Organic Synthesis: Selectivity, Strategy and Efficiency in Modern Organic Chemistry (edited by B.M. Trost and lan Fleming). The volumes are organized according to the bonds being formed or broken - creating carbon-carbon $\sigma$ linkages, for example, or adding to $\mathrm{C}-\mathrm{C} \pi$ bonds. As mechanistic details are provided for many of the reactions, the series is likely to be a useful reference source for research as well as an exhaustive organic 'cookbook'. Published by Pergamon Press, the complete set, including cumulative indexes, is priced at $£ 1,950, \$ 3,900$.

P.B.

\section{Thinly spread}

Carl V. Thompson

The Materials Science of Thin Films, By Milton Ohring. Academic: 1991. Pp. 704. $\$ 69.95, £ 46.50$.

MAterials science is hot. Advanced materials processing, increasingly recognized as the key to the development of new or improved technologies, has spurred new interdisciplinary research-anddevelopment groups in industry and academic institutions, and plenty of scientists are now working on topics in the traditional area of materials science and engineering.

But many of these researchers have no specific background in materials science, so there is a need for new, comprehensive texts and references in the field and its subdisciplines. This has been especially true for thin films, and of the several recent books on the subject, Milton Ohring's extensive volume is without doubt the best.

Ohring has worked and lectured on thin films for many years. He has drawn on this experience to produce a book that covers a wide range of subjects in a readily accessible way. Like authors of other books on integrated-circuit or thinfilm processing, he deals with the 'unit operations' of processing, including chemical and physical deposition, thin-film reactions, diffusion and lithography. But unlike other authors, Ohring also goes into the technology of film deposition, including diagrams and brief descriptions of the various types of vacuum pumps and evaporation techniques.

Along with the obligatory discussions of the electronic properties of thin films, Ohring includes sections on the magnetic, optical and mechanical properties of films, topics that are usually ignored in other texts. There are also chapters on film characterization and film modification techniques such as ion bombardment and laser annealing. The number of subjects covered is impressive.

But although its encyclopaedic nature is one of the book's strengths, it also contributes to its main flaws. Thin-film research is a fast-moving field, and it would be difficult for anyone to provide a thorough and up-to-date treatment of all its aspects. On any given topic, Ohring often introduces only some of the key concepts, and few areas are dealt with at a depth sufficient to make a related current research paper accessible, although references are often given to more advanced treatments and recent reviews. When there are no such references, however, the reader is left somewhat adrift.

Despite its sometimes superficial treatment, The Materials Science of Thin Films will be a useful introductory textbook and reference work. As a teacher and as a researcher, I shall be glad that it is on my shelf, along with the more advanced literature.

Carl V. Thompson is in the Department of Materials Science and Engineering, Massachusetts Institute of Technology, Cambridge, Massachusetts 02139, USA. 